\title{
Disturbed Connectivity of EEG Functional Networks in Alcoholism: A Graph-Theoretic Analysis
}

\author{
Rui Cao, Zheng Wu, Haifang Li, Jie Xiang and Junjie Chen* \\ College of Computer Science and Technology, Taiyuan University of Technology, Taiyuan, Shanxi, \\ 030024, People's Republic of China
}

\begin{abstract}
Generally, an alcoholic's brain shows explicit damage. However, in cognitive tasks, the correlation between the topological structural changes of the brain networks and the brain damage is still unclear. Scalp electrodes and synchronization likelihood (SL) were applied to the constructions of the EGG functional networks of 28 alcoholics and 28 healthy volunteers. The graph-theoretic analysis showed that in cognitive tasks, compared with the healthy control group, the brain networks of alcoholics had smaller clustering coefficients in $\beta 1$ bands, shorter characteristic path lengths, increased global efficiency, but similar small-world properties. The abnormal topological structure of the alcoholics may be related to the local-function brain damage and the compensation mechanism adopted to complete tasks. This conclusion provides a new perspective for alcoholrelated brain damage.
\end{abstract}

Keywords: alcoholic, EEG, brain functional network, graph theory

\section{Introduction}

Alcoholism has the third largest impact on human health and physical ability [1]. Chronic drinking can lead to alcohol-related brain damage (ARBD), including brain structure changes, which show up as grey matter change [2], white matter deficits [3], mild brain atrophy [4], frontal system damage [3], corpus callosum atrophy [5], and brain cognitive function damage [6] which includes working memory deficits [7], executive function damage [8], a weakened capacity for processing complex information [9] and an impaired sense of smell [6]. Moreover, chronic alcohol abuse can cause malnutrition and liver dysfunction, which can result in serious diseases, such as Wernicke encephalopathy and Korsakoff syndrome [4].

EEG technology can record the electrical activity of the brain non-invasively with high temporal resolution, and it is convenient to collect and inexpensive. Thus, it has been widely used in the studies of pathological mechanisms, and clinical manifestations of diverse neurological diseases (e.g.

\footnotetext{
*Corresponding author. Junjie Chen, College of Computer Science and Technology (Taiyuan University of Technology), Taiyuan 030024, Shanxi Province, China. Tel.: 03516010071; E-mail: chenjunjie_tyut@ sina.com.
}

0959-2989/14/\$27.50 @ 2014 - IOS Press and the authors. 
epilepsy) [10], mental disorder (e.g. depression, ADHD) [11], and neurodegenerative disease (for example, Alzheimer's disease and ARBD) [12], meanwhile, in the research of different cognitive functions [13]. Generally, quantitative analysis methods of EEG data primarily include time-domain analysis, frequency-domain analysis, time-frequency analysis, and the nonlinear dynamics theory. A new research approach involves using the complex network method to analyze the EEG data.

The human brain is a highly complex structural network consisting of numerous neuronal cells that are connected through synapses. Based on the physical structure of the brain, spontaneous activities of neuronal cells and stimulated synaptic transmissions make neural activities between neuronal cells and the nervous system coordinated, and together form a complex functional network [14]. Therefore, using the complex network theory to study the topological structure of the structural network and functional network can reveal the structural and functional changes of the brain under disease or cognitive tasks, and further understand the pathomechanism and cognitive functions of the brain [15,16]. To date, there has been very little research done on the functional networks in alcoholism. In this study, the EEG functional networks in alcoholic and the control group have been developed and analyzed by graphtheoretic analysis method, which is one of the methods of the complex network theory, to reveal the topological changes of the functional network of the brain.

\section{Data and method}

\subsection{Data}

All the information in this paper comes from the data set of the University of California, Irvine, used for machine learning. This original data set recorded the multi-channel EEG time sequences of two groups, which included 77 alcoholics and 45 participants of the control group. In this experiment, the data of channe-61 scalp EEG in two sets of volunteers was collected, and the electrode position was based on the 10-20 system of international standard, the sampling set at $256 \mathrm{~Hz}$, using the modified working-memory task experimental paradigm. Every participant did 120 experiment tasks, and every task was randomly chosen from three different experimental conditions, including single stimulation, double matching stimulation and double nonmatching stimulation. Single stimulation (S1) means presenting only one picture, double matching stimulation (S2 Match) refers to presenting two identical pictures within a short time, and double no-matching stimulation (S2 No Match) means presenting two different pictures within a short time. Moreover, no answer was needed for single stimulation, but the participants under double stimulations were required to judge whether the two figures were the same and press buttons to reply. More specific details about the participants and data description are shown in [17]. In this study, the data of 28 alcoholics and 28 healthy volunteers under three experimental conditions (S1, S2 Match, and S2 No Match) for eight seconds (2048 time points in total) were chosen for further analysis, since the data was incomplete due to lack of concentration and wrong replies given by volunteers. In addition, mastoid reference electrodes focus on the long-distance connections at the expense of small scale details [18], so the reference electrode was transferred to an average reference electrode and ocular artifacts were removed.

\subsection{Wavelet packet decomposition}

In the human brain, different neuron oscillation frequencies have a close correlation with the functional activities of the brain [19]. Wavelet analysis is a usually used method in decomposing of signal frequency. 
Considering the deficient resolution of wavelet decomposition (WD) in high frequency band, we used wavelet packet decomposition (WPD). WPD is efficient than WD for frequency decomposing, and it can be further decomposed high frequency signal as well as low frequency signal. Here, the EEG signals are decomposed into 7 levels, using Daubechies $4(\mathrm{db} 4)$ for mother wavelet to distinguish the usable signal. All of EEG data was decomposed into five frequency bands: $\delta(0-4 \mathrm{~Hz}), \theta(4-7 \mathrm{~Hz}), \alpha(7-13 \mathrm{~Hz})$, $\beta 1(13-20 \mathrm{~Hz})$, and $\beta 2(20-30 \mathrm{~Hz})$.

\subsection{Network construction}

Network construction primarily chooses the nodes and edges. In this case, scalp electrodes were chosen as the nodes, and there were 61 nodes in total. The correlation intensity between the nodes, which is the dependencies between the time sequences of the two electrodes, was usually measured by the synchronization. Since the cognitive activities of the brain are based on coordination between several nervous systems or brain areas, the quantitative analysis of the synchronization can provide some proof for the research on functional brain integration [20]. Herein, synchronization likelihood (SL) was used to evaluate the interdependence between two nodes [21] and the calculation parameters were chosen by time-frequency characteristics of the data [22]. In fact, SL, used for measuring the linear and nonlinear interdependence between two time serials, is one of the measurements for generalized synchronization, and it has been widely applied in the studies of EEG brain networks because of good noise immunity and excellent show in non-stationary data. The $61 * 61$ correlation matrix $\mathrm{R}$ can be achieved by the calculation of SL between every pair of nodes. The elements $\mathbf{R}_{i j}$ in the matrix represent the dependent intensity of the two corresponding nodes. Choosing an appropriate threshold $\mathrm{T}$ and binarizing the correlation matrix results in the adjacency matrix $\mathrm{A}$. If $\mathrm{R}_{i j} \geqslant \mathrm{~T}$, then $\mathrm{A}_{i j}=1$, which means the corresponding two nodes have an edge. Otherwise, $\mathrm{A}_{i j}$ equals 0 , indicating there is no edge between the two nodes. Therefore, different threshold values lead to different numbers of edges, and influence the metrics of the network [23,24]. To compare the pure differences of the network topological structures, the comparison of complex networks should be under equal numbers of nodes and edges [25].

Density, the linked number in the binary matrix divided by the maximum possible linked number, was used to confirm the number of network edges. To exclude the influence of single density on the network property, larger scale densities are used and the network properties under each density have been compared. It has been proved that the human brain is a typical highly-efficient, low-consumption small-world network [26]. Therefore, small-world characteristic, the basic property of the brain network, can be used to confirm the scale of density. So fake linkage can be removed maximum possibly while small world property is guaranteed according to following method [27,28]. The rules specify that: (1) The average degree of the constructed network should be bigger than $2 \times \log \mathrm{N}$, where $\mathrm{N}$ means the number of nodes; and (2) The small-world property $\sigma$ of the constructed network of all subjects should be bigger than 1.2. According to these rules, in this study, the scale of density is $12-40 \%$, and the brain networks of every density are constructed with a $2 \%$ increasing step-size.

\subsection{Network metrics}

Graph theory is one of the most important mathematical tools used to analyze complex networks. In this theory, a complex network is described as a graph. Graph G (V, E) consists of the set of nodes V and edges E. If any pair of nodes $(\boldsymbol{i}, \boldsymbol{j})$ and $(\boldsymbol{j}, \boldsymbol{i})$ corresponds to the same edge, then the network is an undirected network; otherwise it is a directed network. If every edge is given a weight, then the network 
is a weighted network; otherwise it is called an unweighted network. The EEG network constructed here is an undirected and unweighted network. In a graph theory for analyzing the EEG network, common network metrics include clustering coefficient, characteristic path length, global efficiency and local efficiency. These attributes describe properties of the brain network in functional segregation (clustering coefficient and local efficiency) and functional integration (average path length and global efficiency) [15]. In contrast, small-world index can measure both the segregation and the integration of the network. The small-world network indicates a higher clustering coefficient and shorter path length, and combines the topological advantages of both random and regular networks, so it guarantees the high efficiency of message delivery on a global and local level [14].

The clustering coefficient of node $\boldsymbol{i}$ is defined as the existing edges between neighbor nodes (linked by a direct edge) divided by the maximum possible number of edges between neighbor nodes, as shown in Formula (1):

$$
\mathbf{C}_{i}=\mathbf{E}_{i} / \frac{1}{2} \mathbf{k}_{i}\left(\mathbf{k}_{i}+1\right)
$$

Here $\mathbf{k}_{i}$ is the number of neighbor nodes of node $\boldsymbol{i}, \mathbf{E}_{i}$ is the existing number of edges between $\mathbf{k}_{i}$ nodes, and the clustering coefficient of the whole network $(\mathbf{C})$ is the average of the clustering coefficients of all nodes.

The characteristic path length $(\mathbf{L})$ is defined as the average of the shortest path of any two nodes in a network as shown in Formula (2):

$$
\mathbf{L}=\sum_{i \geqslant j} \mathbf{L}_{i j} / \frac{1}{2} \mathbf{N}(\mathbf{N}+1)
$$

Here $\mathbf{N}$ is the number of nodes, $\mathbf{L}_{i j}$ is the shortest path length of two nodes $\boldsymbol{i}$ and $\boldsymbol{j}$.

The concept of global efficiency is brought up because the disconnected nodes in the network lead to infinite shortest path length [29], so the global efficiency $\left(\mathbf{E}_{\mathbf{g}}\right)$, which is defined as the inverse of the harmonic mean of the shortest path length between each pair of nodes, is the comprehensive indicator of the message transfer delivery speed in a network, and it can be calculated by the Formula (3) as shown below:

$$
\mathbf{E}_{\text {global }}=\frac{1}{\mathbf{N}(\mathbf{N}-1)} \sum_{i \neq j} \frac{1}{\mathbf{L}_{i j}}
$$

The small-world property $\sigma$ is quantified under the base of the random network [30], as illustrated in Formula (4), where $\mathbf{C}_{\text {random }}$ and $\mathbf{L}_{\text {random }}$ represent the clustering coefficient and characteristic path length of the random network, respectively. In this study, for every density, 50 random networks were derived and the average of $\mathbf{C}$ and $\mathbf{L}$ was calculated for $\mathbf{C}_{\text {random }}$ and $\mathbf{L}_{\text {random, respectively. When } \sigma \text { is }}$ much bigger than one, it indicates that the network is a small-world one, and the bigger the value is, the stronger the small-world property is.

$$
\sigma=\frac{\mathbf{C} / \mathbf{C}_{\text {random }}}{\mathbf{L} / \mathbf{L}_{\text {random }}}
$$


A

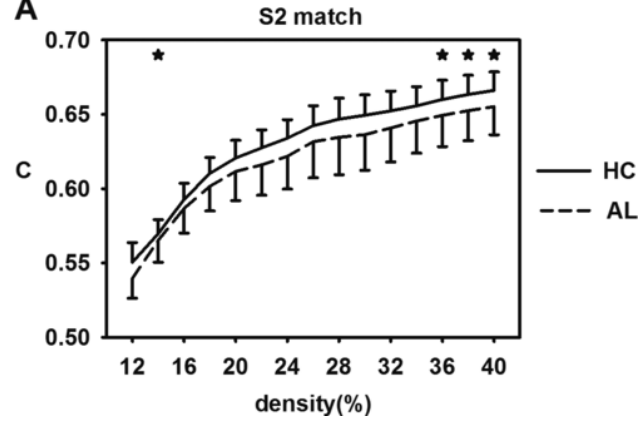

B

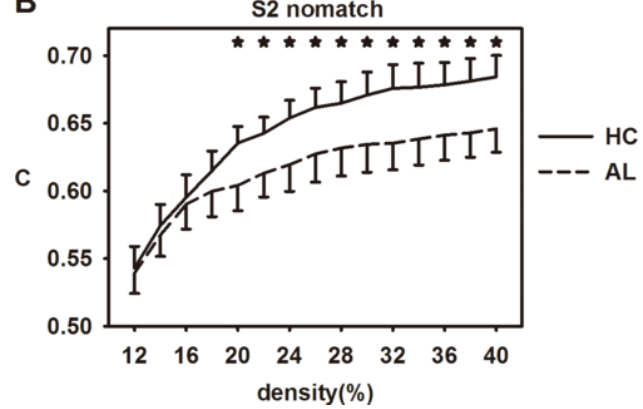

Fig. 1. Clustering Coefficient C of the Brain Networks in Two Groups under Different Densities. (A) Shows S2 Match Tasks; (B) Shows S2 No Match Tasks.
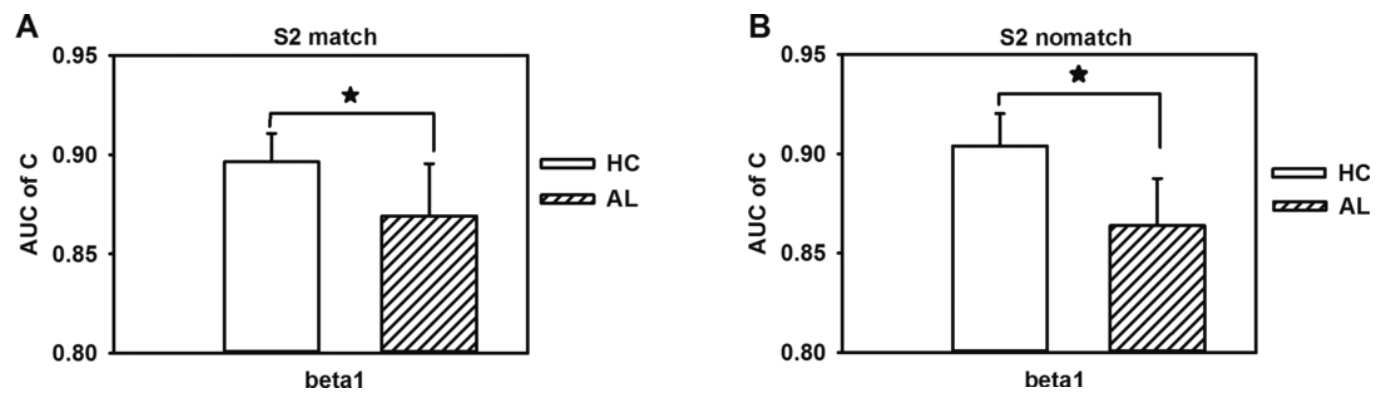

Fig. 2. AUC of Clustering Coefficient in Two Groups, where (A) Represents S2 Match Tasks and (B) Shows S2 No Match Tasks.

\section{Results}

\subsection{Clustering coefficient}

It has been proposed that the clustering coefficient $\mathbf{C}$ in the brain networks of the alcoholics and the control group changes over different densities. As shown in Figure 1, within the density scale of $12-40 \%$, when the density is enhanced (an increased number of edges in the network), $\mathbf{C}$ in the two categories showed monotonous increasing trends. In the S2 Match tasks, the clustering coefficients $\mathbf{C}$ of the brain networks in the alcoholics group and the healthy control group are clearly dissimilar under various densities ( $p<0.05, \mathrm{KS}$ test). This is also true for C in the S2 No Match tests, which revealed an even bigger range of the densities. All the results mentioned to this point show only in the $\beta 1$ bands. On a side note, in all the figures in this paper, $\mathrm{HC}$ means healthy control, AL means alcoholic, error lines represent $95 \%$ confidence interval, and $\star$ means that there exists significant differences between two groups $(p<0.05$, KS test).

To characterize the differences of the network property in the entire density range instead of relying on a single threshold, the area under the curve (AUC) of the network property, which is sensitive to the changes of network topological property [28], was introduced and calculated. Since the evident difference of $\mathbf{C}$ in two groups only exists in $\beta 1$ bands, the AUC analysis was performed in this area under the increasing size-step of $2 \%$ within the density range of $12-40 \%$, and tested by KS. As illustrated in Figure 2 , the $\mathrm{C}$ of the alcoholics is obviously smaller than that of the healthy control group, both in S2 Match tasks, $(p=0.021019, \mathrm{KS}$-test $)$ and S2 No Match tasks ( $p=0.003842, \mathrm{KS}$-test $)$. 
A

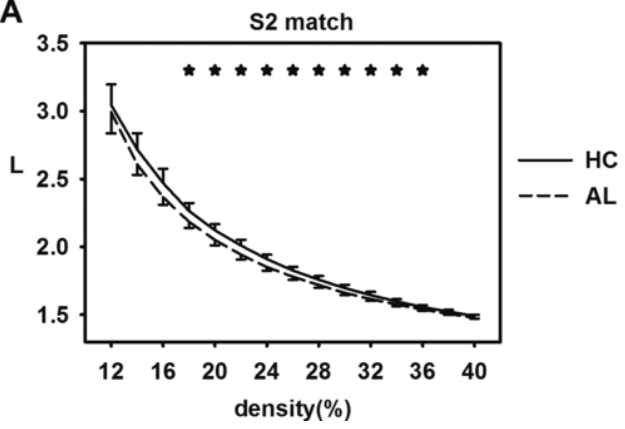

B

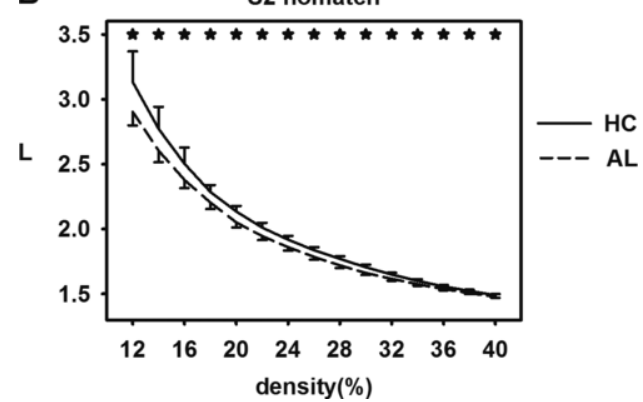

Fig. 3. Characteristic Path Lengths of the Brain Networks in Two Groups under Different Densities. (A) Represents S2 Match Tasks; (B) Shows S2 No Match Tasks.

A

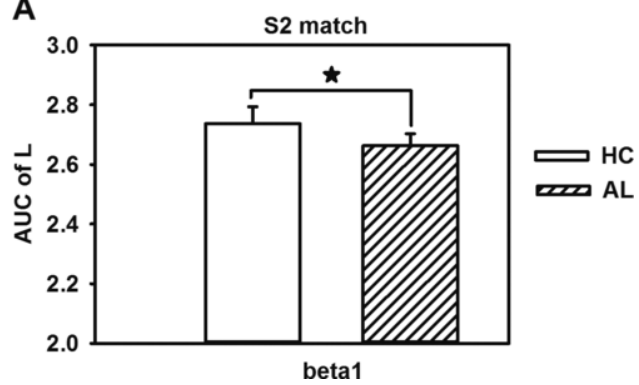

B

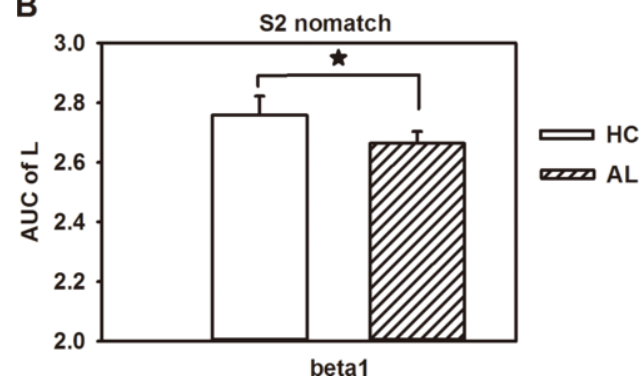

Fig. 4. AUC of Characteristic Path Lengths in Two Groups, where (A) Represents S2 Match Tasks and (B) Shows S2 No Match Tasks

\subsection{Characteristic path length}

As shown in Figure 3, all the characteristic path lengths of the two groups in the different densities are significantly different. The evident difference of the two volunteer groups was in the density range of $18-36 \%$ for the S2 Match tasks ( $p<0.05, \mathrm{KS}$-test). As for the S2 No Match tasks, it was in the range of $12-40 \%$ ( $p<0.05, \mathrm{KS}$-test). Similar to the results of $\mathbf{C}$, all these differences exist only within the $\beta 1$ bands.

The AUC test results for the characteristic path lengths $\mathbf{L}$ of the two volunteer groups in $12-40 \%$ density within the $\beta 1$ bands are shown in Figure 4 . The AUC values of the characteristic path lengths $\mathbf{L}$ for the alcoholics are much smaller than the healthy control group both in S2 Match $(p=0.021019$, KS-test) and S2 No Match tasks ( $p=0.000168, \mathrm{KS}$-test).

\subsection{Global efficiency}

Since the global efficiency $\mathbf{E}_{\mathbf{g}}$ is the harmonic inverse average of the characteristic path length, the $\mathbf{E}_{\mathbf{g}}$ values of the two groups is increasingly monotonous, and the evident difference between the two groups shows up in the same range of density as the one in the characteristic path length within $\beta 1$ bands, as shown in Figure 5. More specifically, the significant differences of $\mathbf{E}_{\mathbf{g}}$ values of the two groups exist in every density in the range of $18-36 \%$ for S2 Match tasks $(P<0.05$, KS-test $)$ and $12-40 \%$ for S2 No Match tasks $(P<0.05, \mathrm{KS}$-test $)$. 
A

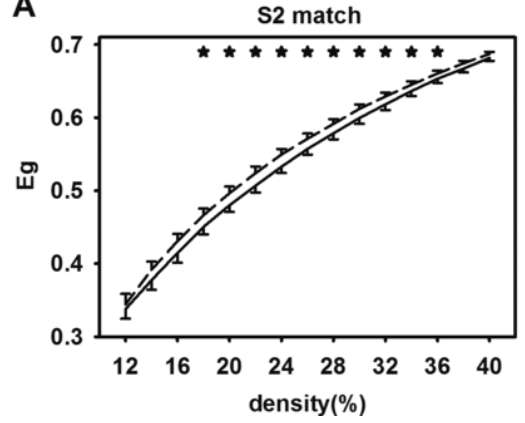

B

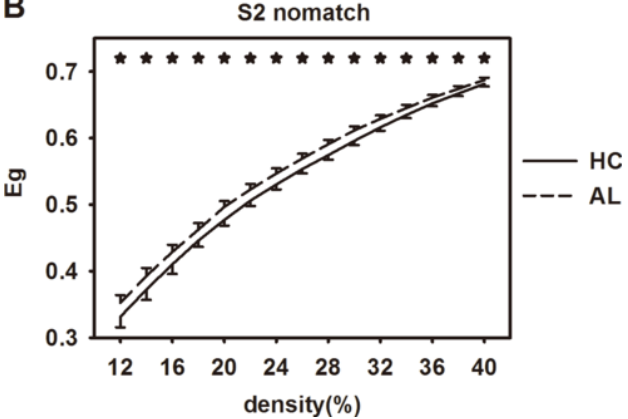

Fig. 5. Global Efficiency $\mathrm{E}_{g}$ of the Brain Networks in Two Groups under Different Densities. (A) Represents S2 Match Tasks; (B) Shows S2 No Match Tasks
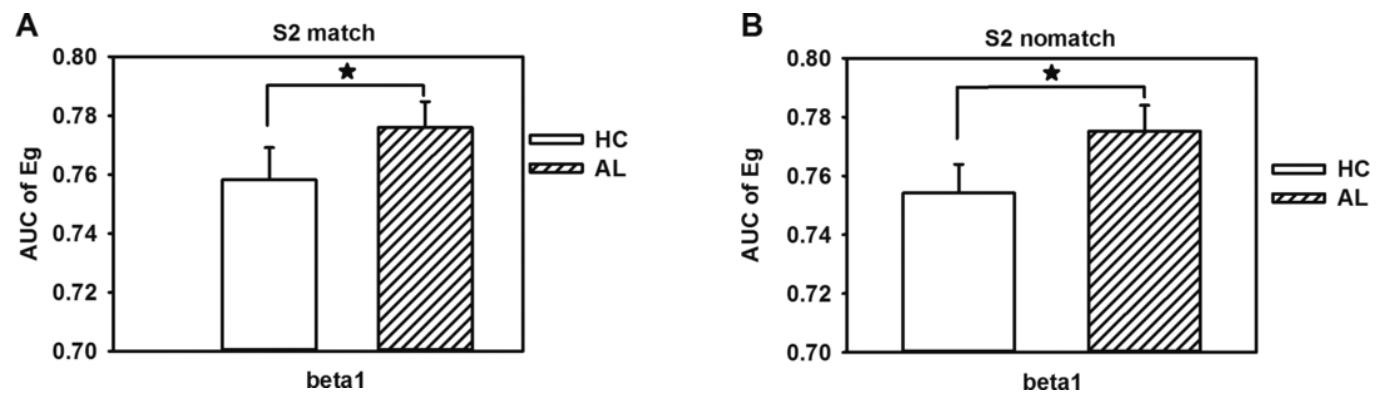

Fig. 6. AUC of Global Efficiency in Two Groups, where (A) Represents S2 Match tasks and (B) Shows S2 No Match tasks.

As shown in Figure 6, within $\beta 1$ bands, the AUC values for the global efficiency of the alcoholics in the density of $12-40 \%$ are significantly higher than that of the healthy control group, both in S2 Match tasks ( $p=0.00934, \mathrm{KS}$-test) and S2 No Match tasks ( $p=0.00516, \mathrm{KS}$-test $)$.

\subsection{Small-worldness}

The small-worldness $\sigma$ values the topological structure of the network as a whole. As shown in Figure 7 , in $\beta 1$ wave bands, there was no difference between the alcoholics and the healthy control groups in any densities for S2 Match tasks. However, in the S2 No Match tasks, there were slight differences in a small number of densities. In addition, no difference exists in any other bands. Moreover, the AUC test results for the $\sigma$ of the two groups in the densities of $12-40 \%$ are nearly the same.

\section{Discussion}

The main objective of this study was to find the differences in the global properties of functional brain networks of alcoholics and healthy individuals under working memory tasks. The results show that with the increase of density, which refers to the increase of edge amounts, the clustering coefficient $\mathbf{C}$ increases monotonously (Figure 1). However, the AUC test results show that the $\mathbf{C}$ values of the alcoholics in the $12-40 \%$ densities are much smaller than that of the healthy control group, which is in accordance with previous research [31]. The clustering coefficient is the measurement of local linkage. The relatively smaller $\mathbf{C}$ indicates sparse local linkages of brain networks in alcoholics. Many reports reveal that when 
A

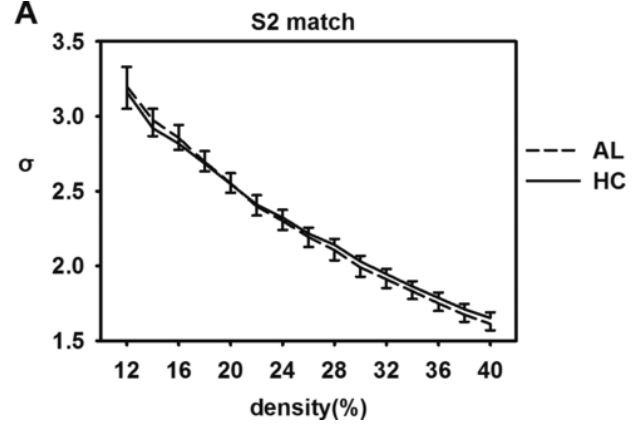

B

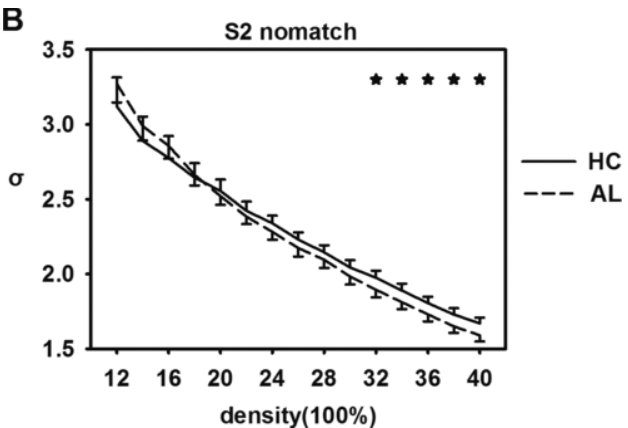

Fig. 7. Small-Worldness $\sigma$ of the Brain Networks in Two Groups under Different Densities ((A) Represents S2 Match tasks; (B) Shows S2 No Match tasks)

alcoholics participate in memory-related tasks, the synchronism between the brain activities in $\alpha$ and $\beta$ bands is weakened [31,32], especially for some areas in the forehead and parietal lobe [33], indicating weakened functional linkages in these regions. In addition, DTI shows that structural components of the white matter in the local brains of alcoholics are damaged, and this influences the working-memory ability [34].

As shown in Figure 3, the characteristic path length $\mathbf{L}$ decreases monotonously with the increase of densities. This is because larger amounts of edges allow more direct links between nodes, resulting in shorter average path lengths. As for the AUC results (Figure 4), the $\mathbf{L}$ of alcoholics in the whole density range is markedly smaller than that of the healthy control group in cognitive tasks, due to longer links between more distant brain areas in the networks of alcoholics. Previous research also found that farther brain area links will exist in the anterior cerebellum systems of alcoholics to guarantee completion of cognitive tasks [7], which indicates that the brain networks of alcoholics tend to randomize their networks. This random network trend is also revealed in other neurodegenerative diseases, such as Alzheimer's [35,36].

The global efficiency $\mathbf{E}_{\mathbf{g}}$ is the harmonic inverse average of the characteristic path length, so the shorter the average path length is, the higher the global efficiency. Therefore, the global efficiency increases monotonously with the increase of density (Figure 5). Moreover, the global efficiency of alcoholics is significantly higher than that of the healthy control group in the S2 tasks as showed in Figure 6. That could be due to local cognitive function damage in alcoholics, they need high-level cognitive systems (more performing systems) to accomplish the same cognitive tasks. This result is powerful proof to the theory that alcoholics try to compensate damaged cognitive ability with high-level cognitive systems [37], which has been proposed in other studies of alcoholics [38,39].

More interestingly, the difference between the groups changes in tasks of different complexity. As shown in Figure 2, the clustering coefficient of alcoholics is much smaller than that of the healthy control group ( $p=0.021019, \mathrm{KS}$ test) in the S2 Match tasks, and this trend gets stronger in the S2 No Match tasks $(p=0.003842, \mathrm{KS}$ test). Similar results are found in the characteristic path lengths and global efficiency (Figure 4,6), where the differences between the two groups in the S2 No Match tasks are greater than those in the S2 Match tasks. Even though the AUC test of small-world-ness $\sigma$ for the two groups was similar, there were evident differences in the two groups under partial densities in the S2 No Match tasks. However, no differences were found in the S2 Match tasks (Figure 7). This may be because the higher complexity of the S2 No Match tasks enlarges the difference between the two groups.

Some valuable findings were revealed in this study on the topological structure changes in the brain function networks of alcoholics, but all these conclusions are based on the EEG data collected when 
subjects were involved in working-memory cognitive tasks. There is still no information available on the performance of brain function networks of alcoholics in a resting state. Further studies on comparing the changes of brain function networks in a resting state and cognitive tasks can be performed in the future to explore the relationship between the topological brain structure of alcoholics and cognitive functions.

\section{Conclusion}

The EEG functional networks of the alcoholics and the healthy control group under cognitive memory tasks have been compared by graph-theoretical analysis. It was found that the topological structure of the brain networks of alcoholics is significantly different from that of healthy controls. Network measurements shows that, in $\beta 1$ bands of $13-20 \mathrm{~Hz}$, the clustering coefficients and characteristic path lengths of brain networks of alcoholics are much smaller than those of healthy controls, but the global efficiency showed the opposite is true. This indicates that compared to the brain networks of the healthy control group, those of the alcoholics have sparse local functional links and an ever-increasing number of longer links. These abnormal topological structures of the alcoholics may be related to local-function brain damage and the compensation mechanisms adopted to perform tasks. This conclusion provides a new perspective for alcohol-related brain damage and the pathological mechanism of related diseases.

\section{Acknowledgement}

This work was financially supported by the National Natural Science Foundation of China (No. 61170136, No. 61373101), Natural Science Foundation of Shanxi (No.2014021022-5) and the Research Fund for the Doctoral Program of Higher Education of China (No. 20131402120009).

\section{References}

[1] W.H. Organization, Global status report on alcohol and health: Department of Mental Health and Substance Abuse (MSD) of the World Health Organization (WHO), Geneva, Switzerland, 2011.

[2] J. Jacobus and S.F. Tapert, Neurotoxic effects of alcohol in adolescence. Annual review of clinical psychology 9 (2013), 703-721.

[3] A. Pfefferbaum, M. Rosenbloom, T. Rohlfing and E.V. Sullivan, Degradation of association and projection white matter systems in alcoholism detected with quantitative fiber tracking. Biol Psychiatry 65 (2009), 680-690.

[4] A.D. Smith, S.M. Smith, C.A. de Jager et al., Homocysteine-lowering by B vitamins slows the rate of accelerated brain atrophy in mild cognitive impairment: a randomized controlled trial. PloS one 5 (2010), e12244.

[5] I.C. Liu, C.H. Chiu, C.J. Chen, L.W. Kuo, Y.C. Lo and W.Y. Tseng, The microstructural integrity of the corpus callosum and associated impulsivity in alcohol dependence: A tractography-based segmentation study using diffusion spectrum imaging. Psychiatry Research: Neuroimaging 184 (2010), 128-134.

[6] C.D. Coles, F.C. Goldstein, M.E. Lynch et al., Memory and brain volume in adults prenatally exposed to alcohol. Brain and cognition 75 (2011), 67-77.

[7] X. Noel, M. Van der Linden, D. Brevers et al., The contribution of executive functions deficits to impaired episodic memory in individuals with alcoholism. Psychiatry research 198 (2012), 116-122.

[8] G.T. Sutherland, P.J. Sheahan, J. Matthews et al., The effects of chronic alcoholism on cell proliferation in the human brain. Experimental neurology 247 (2013), 9-18.

[9] T.G. Alisa Green, S. Donna, B. Helen, A.S. Edwin, and H. Clive, The Effect of Moderate to Heavy Alcohol Consumption on Neuropsychological Performance as Measured by the Repeatable Battery for the Assessment of Neuropsychological Status. Alcoholism: Clinical and Experimental Research 34 (2010), 443-450.

[10] U.R. Acharya, S.S. Vinitha, G. Swapna, R.J. Martis and J.S. Suri, Automated EEG analysis of epilepsy: A review. Knowledge-Based Systems 45 (2013), 147-165. 
[11] D.T. Plante, M.R. Goldstein, E.C. Landsness EC et al., Topographic and sex-related differences in sleep spindles in major depressive disorder: a high-density EEG investigation. Journal of affective disorders 146 (2013), 120-125.

[12] D.F. Hermens, J. Lagopoulos, W.J. Tobias et al., Pathways to alcohol-induced brain impairment in young people: a review. Cortex 49 (2013), 3-17.

[13] J. Xiang, R. Cao and L. Li, Emotion recognition based on the sample entropy of EEG. Bio-medical materials and engineering 24 (2014), 1185-1192.

[14] E.C. van Straaten and C.J. Stam, Structure out of chaos: functional brain network analysis with EEG, MEG, and functional MRI. European neuropsychopharmacology: the journal of the European College of Neuropsychopharmacology 23 (2013), $7-18$.

[15] M. Rubinov and O. Sporns, Complex network measures of brain connectivity: uses and interpretations. Neuroimage 52 (2010), 1059-1069.

[16] O. Sporns, The human connectome: origins and challenges. Neuroimage 80 (2013), 53-61.

[17] G.V. Tcheslavski and F.F. Gonen, Alcoholism-related alterations in spectrum, coherence, and phase synchrony of topical electroencephalogram. Computers in biology and medicine 42 (2012), 394-401.

[18] C. Calmels, M. Foutren and C.J. Stam, Beta functional connectivity modulation during the maintenance of motion information in working memory: importance of the familiarity of the visual context. Neuroscience 212 (2012), 49-58.

[19] D.M. Groppe, S. Bickel, C.J. Keller et al., Dominant frequencies of resting human brain activity as measured by the electrocorticogram. Neuroimage 79 (2013), 223-233.

[20] D.J. Kim, A.R. Bolbecker, J. Howell et al., Disturbed resting state EEG synchronization in bipolar disorder: A graphtheoretic analysis. NeuroImage Clinical 2 (2013), 414-423.

[21] C.J. Stam and B.W. van Dijk, Synchronization likelihood_an unbiased measure of generalized synchronization in multivariate data sets. Physica D 163 (2002), 236-251.

[22] T. Montez, H.K. Linkenkaer, B.W. van Dijk and C.J. Stam, Synchronization likelihood with explicit time-frequency priors. Neuroimage 33 (2006), 1117-1125.

[23] B.C. van Wijk, C.J. Stam and A. Daffertshofer, Comparing Brain Networks of Different Size and Connectivity Density Using Graph Theory. PloS one 5 (2010), e13701.

[24] A. Joudaki, N. Salehi, M. Jalili and M.G. Knyazeva, EEG-based functional brain networks: does the network size matter? PloS one 7 (2012), e35673.

[25] S. Micheloyannis, E. Pachou, C.J. Stam et al., Using graph theoretical analysis of multi channel EEG to evaluate the neural efficiency hypothesis. Neurosci Lett 402 (2006), 273-277.

[26] E. Bullmore and O. Sporns, The economy of brain network organization. Nature reviews Neuroscience 13 (2012), 336349.

[27] S. Achard and E. Bullmore, Efficiency and cost of economical brain functional networks. PLoS computational biology 3 (2007), e17.

[28] J. Zhang, J. Wang, Q. Wu et al., Disrupted brain connectivity networks in drug-naive, first-episode major depressive disorder. Biological psychiatry 70 (2011), 334-342.

[29] V. Latora and M. Marchiori, Efficient Behavior of Small-World Networks. Physical Review Letters 87 (2001), 198701.

[30] M.D. Humphries and K. Gurney, Network 'small-world-ness': a quantitative method for determining canonical network equivalence. PloS one $\mathbf{3}$ (2008), e0002051.

[31] V. Sakkalis, T. Oikonomou, V. Tsiaras and I. Tollis, Graph-theoretic Indices of Evaluating Brain Network Synchronization: Application in an Alcoholism Paradigm. Neuromethods 88 (2013).

[32] E.A. de Bruin, C.J. Stam, S. Bijl, M.N. Verbaten and J.L. Kenemans, Moderate-to-heavy alcohol intake is associated with differences in synchronization of brain activity during rest and mental rehearsal. International journal of psychophysiology: official journal of the International Organization of Psychophysiology 60 (2006), 304-314.

[33] R.R. Wetherill, S. Bava, W.K. Thompson et al., Frontoparietal connectivity in substance-naive youth with and without a family history of alcoholism. Brain research 1432 (2012), 66-73.

[34] A. Pfefferbaum, E. Adalsteinsson and E.V. Sullivan, Dysmorphology and microstructural degradation of the corpus callosum: Interaction of age and alcoholism. Neurobiology of aging 27 (2006), 994-1009.

[35] Y. He and A. Evans, Graph theoretical modeling of brain connectivity. Current opinion in neurology 23 (2010), 341-350.

[36] C.J. Stam, W. de Haan, A. Daffertshofer et al., Graph theoretical analysis of magnetoencephalographic functional connectivity in Alzheimer's disease. Brain: a journal of neurology 132 (2009), 213-224.

[37] A.L. Pitel, T. Witkowski, F. Vabret, B. Guillery-Girard et al., Effect of episodic and working memory impairements on semantic and cognitive procedural learning at alcohol treatment entry. Alcoholis:, clinical and experimental research $\mathbf{3 1}$ (2007), 238-248.

[38] S. Chanraud, A.L. Pitel, A. Pfefferbaum and E.V. Sullivan, Disruption of functional connectivity of the default-mode network in alcoholism. Cerebral cortex 21 (2011), 2272-2281.

[39] S. Chanraud, A.L. Pitel, E.M. Muller-Oehring, A. Pfefferbaum and E.V. Sullivan, Remapping the brain to compensate for impairment in recovering alcoholics. Cerebral cortex 23 (2013), 97-104. 